\title{
Structural equation modeling of associations among combat exposure, PTSD symptom factors, and Global Assessment of Functioning
}

\author{
Mark W. Miller, PhD; ${ }^{*}$ Erika J. Wolf, MA; Elaine Martin, BS; Danny G. Kaloupek, PhD; Terence M. Keane, PhD \\ National Center for Posttraumatic Stress Disorder, Department of Veterans Affairs Boston Healthcare System, Boston, MA; \\ Boston University School of Medicine, Boston, MA
}

\begin{abstract}
This study used structural equation modeling (SEM) to evaluate the relative strength of associations between combat exposure, four posttraumatic stress disorder (PTSD) symptom factors, and functional impairment as indexed by Global Assessment of Functioning (GAF) scores in a sample of 315 veterans assessed at a Department of Veterans Affairs PTSD clinic. Results showed that the association between combat exposure and GAF scores was fully mediated by PTSD severity. The bestfitting model included direct paths from combat exposure to reexperiencing symptoms and from avoidance and numbing symptoms to GAF. However, only $17 \%$ of variance in GAF was accounted for by PTSD. The results raise concern about the use of the GAF score as a benchmark for quantifying combat PTSDrelated functional impairment.
\end{abstract}

Key words: avoidance, combat exposure, functional impairment, Global Assessment of Functioning, hyperarousal, numbing, PTSD, reexperiencing, rehabilitation, structural equation modeling.

\section{INTRODUCTION}

Posttraumatic stress disorder (PTSD) is a major health concern for military war veterans and the Department of Veterans Affairs (VA). Estimates based on the National Vietnam Veterans Readjustment Study (NVVRS) indicate that between 9 and 15 percent of male Vietnam veterans met diagnostic criteria for current PTSD approximately 20 years after the Vietnam war and that between 19 and 31 percent of male veterans had met criteria for the disorder at some point since the Vietnam war [1-2]. More recently, the mental health of veterans of conflicts in Iraq and Afghanistan has become a national priority. In one recent study of troops returning from Iraq, Hoge et al. found that approximately 15 percent met presumptive criteria for PTSD, major depression, and/or generalized anxiety disorder and of those who met criteria for one of these disorders, 75 percent had PTSD [3].

PTSD exacts an enormous and growing cost from American society and the VA. A recent Inspector General's report found that the number of veterans receiving disability awards for PTSD, as well as the size of those awards, is growing [4]. From 1999 to 2004, the total number of PTSD cases increased by 79.5 percent while PTSD benefits payments grew 148.8 percent from $\$ 1.7$ billion to

\footnotetext{
Abbreviations: C\&P $=$ Compensation and Pension, CAPS = Clinician-Administered PTSD Scale, CES = Combat Exposure Scale, $\mathrm{CFA}=$ confirmatory factor analysis, $\mathrm{CFI}=$ comparative fit index, DSM-IV = Diagnostic and Statistical Manual of Mental Disorders-Fourth Edition, GAF = Global Assessment of Functioning, NVVRS = National Vietnam Veterans Readjustment Study, PTSD = posttraumatic stress disorder, SD = standard deviation, SEM = structural equation modeling, SRMR = standardized root-mean-square residual, TLI = Tucker-Lewis index, VA = Department of Veterans Affairs, VHA = Veterans Health Administration .

*Address all correspondence to Mark W. Miller, PhD; National Center for PTSD (116B-2), VA Boston Healthcare System, 150 South Huntington Ave, Boston, MA 02130; 857364-5733; fax: 857-364-4501. Email: Mark.Miller5@va.gov DOI: 10.1682/JRRD.2007.06.0085
} 
$\$ 4.3$ billion. Furthermore, while veterans being compensated for PTSD represented only 8.7 percent of all compensation recipients, this subgroup received 20.5 percent of all compensation payments. The VA Compensation and Pension (C\&P) determination process on which these awards are based involves a review of the veteran's military and medical record; assessment of trauma exposure, PTSD, and mental status; evaluation of social and occupational function; and assignment of a Global Assessment of Functioning (GAF) score [5]. The latter is a numerical rating of the overall severity of psychiatric disturbance and is assigned by a clinician. In cases in which a GAF score is unavailable, C\&P raters may request that an evaluator provide one and the Board of Veterans' Appeal may also require a GAF score as part of a remand of a rating decision [6]. The central role of the GAF score in VA mental health assessment is further evidenced by Veterans Health Administration (VHA) Directive 97-059, which mandated that clinicians record "at least one GAF score reflecting the 'current level of functioning' for each veteran patient seen at any VHA mental health inpatient or outpatient setting" [7].

Despite the VA's adoption of the GAF as a benchmark for the assessment of PTSD-related and other psychiatric impairment, concerns have been raised about the scale's reliability, validity, and suitability for this purpose. Criticisms have focused on the use of one item to measure many different functional areas, limitations due to the origin of the GAF as a measure for the study of affective disorders and schizophrenia, and evidence that the score may have a greater association with psychiatric symptoms than with functional abilities [6,8]. Another concern is that because the assessment of functional impairment is involved in the determination of PTSD severity, both at the individual symptom level and the syndrome level, an inherent overlap, or confound, exists between ratings of GAF and PTSD severity. Estimating the strength of this association (i.e., degree of overlap) in a veteran sample with PTSD was a primary aim of this study.

Prior research on the relationship of GAF scores to PTSD severity in such samples is surprisingly limited given its role in VA assessment. One large-scale study by Greenberg and Rosenheck that did examine the utility of the GAF as a VA mental health outcome measure found a moderate association between the PTSD diagnosis and GAF in outpatients [9]. The authors concluded that the GAF was reasonably reliable, discriminated severe illnesses such as schizophrenia and Alzheimer disease from those of lesser severity, and was sensitive to improvement. In a study of veterans receiving services at a VA PTSD clinic, Miller et al. reported the reliability of GAF ratings assessed by two different raters within 30 days (e.g., a combined test-retest and interrater reliability index) to be 0.55 [10]. These investigators also reported a significant negative association between the severity of posttraumatic psychopathology and GAF scores. Similarly, in another study, Miller et al. reported an interrater reliability coefficient of 0.62 for the GAF scale in a large sample of Vietnam veterans with PTSD and reported lower GAF scores among participants with complex versus simple forms of posttraumatic psychopathology [11]. To our knowledge, however, no prior study has examined the multivariate relationships that may exist between the severity of military-related trauma (i.e., combat exposure), PTSD severity, and current GAF in a VA treatment-seeking sample of combat veterans.

\section{Factor Structure of PTSD and Its Relationship to Trauma Exposure and Functional Impairment}

PTSD is defined by 17 symptoms that reflect profound disturbances in cognitive, affective, behavioral, and physiological functioning. The Diagnostic and Statistical Manual of Mental Disorders-Fourth Edition (DSM-IV) groups these symptoms into three clusters: reexperiencing of the event (e.g., recurrent and intrusive thoughts, distressing dreams), avoidance and emotional numbing (e.g., avoidance of reminders of the traumatic event, restricted range of affect), and hyperarousal (e.g., hypervigilance, sleep difficulties) [5]. However, this organization of symptoms, specifically the grouping together of avoidance and numbing symptoms, has received little support from factor analytic studies. To the contrary, when clinicians have used structured diagnostic interviews to assess PTSD, most studies have provided support for a four-factor model that disaggregates the effortful avoidance and numbing symptoms [12-13].

Our first aim was to examine the differential relationships between these four symptom factors (reexperiencing, avoidance, numbing, and hyperarousal) and the etiologic factor believed to be primarily responsible for the development of the syndrome in this sample: combat exposure. We hypothesized that the reexperiencing and avoidance symptoms would show the strongest relationship with combat exposure severity. Extant theory and research provide several bases for this prediction. First, reexperiencing and avoidance symptoms are, by definition, thematically linked to the traumatic event and have long been considered the cardinal symptoms of the disorder. Second, individuals who endure more extensive traumas would be expected to have more extensive networks of traumatic memory, which 
increase the potential for traumatic memories to be retrieved and more extensively activated. Third, avoidance symptoms are functionally intertwined with reexperiencing, because they minimize exposure to trauma-related stimuli and might therefore be expected to show a comparable association with trauma severity. Fourth, research suggests that the reexperiencing and avoidance symptoms have superior discriminant validity as markers of posttraumatic psychopathology compared with the numbing and hyperarousal symptoms, which overlap with the general distress and dysphoria common to all anxiety and mood disorders [14-15]. Consistent with these postulates, prior investigations have found stronger associations between trauma exposure and reexperiencing/avoidance symptoms than with numbing and hyperarousal symptoms [16-17]. In contrast, the numbing and hyperarousal symptoms have no thematic relationship to the traumatic event, do not explicitly involve traumatic memories or responses to trauma-related stimuli, and comprise primarily non-trauma-specific symptoms of psychiatric disturbance.

Our second aim was to examine the hypothesis that the association between combat exposure and functional impairment is mediated by PTSD. Mediation models seek to identify mechanisms that underlie observed relationships between an independent variable (e.g., combat exposure) and a dependent variable (e.g., GAF scores) via the inclusion of mediating variables in the equation (e.g., PTSD factors). The NVVRS [1] and other studies of combat veterans have repeatedly demonstrated associations between (1) combat exposure and functional impairment, (2) combat exposure and PTSD, and (3) PTSD and functional impairment, but to our knowledge, no prior study has attempted to model the mediating influences of each of the four PTSD factors on the association between combat exposure and GAF scores. This study addressed this gap in our knowledge base.

Prior studies that have examined the association between the four PTSD symptom clusters and outcome measures related to functional impairment have yielded mixed results. For example, some studies have found that the numbing and hyperarousal symptoms are the strongest predictors of outcomes such as self-reported physical health functioning [18-20] and PTSD chronicity [21]. Others have shown the numbing and avoidance symptoms to be uniquely associated with interference with activities, dissatisfaction with work performance, and social dysfunction [22-23]. Another body of research has underscored the role of emotional numbing in negative life outcomes such as poor general life satisfaction [19] and resiliency resource loss [24]. Still other studies have found unique associations between reexperiencing symptoms and deficits in the physical health domain [25-26]. To summarize, although mixed, results of prior studies of the association between specific PTSD symptom factors and functional impairment implicate emotional numbing as the factor that accounts for the most variance in measures of functional impairment.

\section{Present Study}

In this study, we used structural equation modeling (SEM) to evaluate the relative strength of associations between combat exposure, four PTSD symptom factors, and GAF-rated impairment in a sample of veterans assessed at the beginning of treatment in a VA PTSD clinic. Based on the foregoing literature review, our primary hypotheses were as follows:

1. The effect of combat exposure on GAF would be fully mediated by PTSD.

2. The strength of the association between combat exposure and both reexperiencing and avoidance symptoms would be greater than the association between combat exposure and both numbing and hyperarousal symptoms.

3. Of the four PTSD symptom factors, emotional numbing would show the strongest association with GAF scores.

\section{METHODS}

\section{Participants}

Participants were 315 male combat veterans who underwent diagnostic assessment for PTSD at a VA outpatient clinic between 1996 and 2006. All assessments were voluntary and conducted at the request of the veteran or the veteran's mental or medical healthcare provider. All participants experienced a combat-related traumatic event meeting the DSM-IV PTSD definition for Criterion A, and all met diagnostic criteria for the disorder as assessed by clinicians using the ClinicianAdministered PTSD Scale (CAPS) [27-28].

The mean \pm standard deviation (SD) age of the sample at the time of diagnostic interview was $55 \pm 10$ years (range 24-84). Among participants, 81 percent identified themselves as Caucasian, 13 percent as Black, and 3 percent as Hispanic. The majority of the sample was either working full-time (37\%), retired or disabled (30\%), or unemployed (20\%), with a smaller portion working part-time (8\%). Regarding education, 13 percent did not graduate from high school, 15 percent had received a high school diploma 
or general equivalency diploma, 48 percent had received some college education, and 24 percent had received a bachelor's degree or higher. Study participants served in combat theaters in Vietnam (76\%), Korea (4\%), World War II (6\%), Operation Desert Storm (8\%), Operations Iraqi and Enduring Freedom (1\%), and other locations (5\%). Sixty-two percent served in the Army, seven percent in the Air Force, eight percent in the Navy, and twentythree percent in the Marines. The majority of participants (57\%) reported having a medical and/or psychiatric VA service-connected disability, and 37 percent of those who were service-connected were receiving compensation for PTSD.

\section{Procedures}

Analyses were based on archival clinical data collected during a standard PTSD assessment that took place over several weeks. Doctoral-level clinical psychologists, predoctoral clinical psychology interns, or graduate practicum students conducted the assessments. Unlicensed clinicians were supervised by licensed psychologists. Veterans were interviewed to determine the nature of their Criterion A event(s). All participants in this study reported a combatrelated trauma. After the trauma-exposure assessment, veterans were interviewed with the CAPS (details follow). Subsequently, on a separate visit, veterans completed selfreport measures, including the Combat Exposure Scale (CES) [29]. Finally, clinicians wrote an integrated report of their assessment findings, including a multiaxial diagnosis and current GAF score reflecting overall functioning at the time of the assessment.

\section{Measures}

\section{Clinician-Administered PTSD Scale}

The CAPS is the current gold standard clinicianadministered semistructured interview for PTSD assessment [27-28]. Clinicians rate the frequency and intensity of each of the 17 key symptoms and associated features of PTSD as defined in DSM-IV. The frequency and intensity ratings both range from 0 to 4 and can be summed for a severity score for each symptom. The CAPS possesses strong psychometric properties, including good sensitivity and specificity [28,30]. PTSD diagnostic status (and study eligibility) was determined by a validated scoring rule [31] that required participants to have at least one reexperiencing symptom, three avoidance and numbing symptoms, and two hyperarousal symptoms, each with a frequency score of $\geq 1$ and an intensity score of $\geq 2$. The overall internal consistency (Cronbach $\alpha$ ) for the 17 symptoms was
0.77, and the mean internal consistency for the four symptom factors was 0.56 (range $=0.42-0.69$ ).

\section{Global Assessment of Functioning}

The GAF is a composite rating of overall functioning (psychological, social, and occupational) made by a clinician [5]. It constitutes Axis V of the DSM-IV and ranges from 1 ("persistent danger of severely hurting self or others") to 100 ("superior functioning”). Behavioral, occupational, and social anchors are provided at each 10-point increment along the scale. In this sample, GAF scores ranged from 20 to 75 , with a mean \pm SD of $47 \pm 9$.

\section{Combat Exposure Scale}

We used the CES to assess, on a 1 to 5 ordinal scale, the number of times each participant was exposed to each of seven types of combat experiences [29]. Prior research has shown the CES to be highly reliable (Cronbach $\alpha=0.85$, test-retest reliability $=0.97$ ) and a consistent predictor of PTSD symptomatology and diagnostic status in veterans [29]. In this sample, the internal consistency coefficient for the CES was 0.87. CES raw scores ranged from 7 to 35, with a mean of 22.51, which corresponds to a moderate level of combat intensity (CES mean scaled score $=23.94$ ).

\section{Overview of Structural Equation Modeling}

The analyses used in this study were based on confirmatory factor analysis (CFA) and SEM. Both CFA and SEM are multivariate statistical procedures that permit the modeling of common factors underlying multiple indicators of a construct and measurement error, rather than using only observed or measured variables in their analyses. One strength of this approach is that it quantifies the amount of variance in indicators due to the latent construct versus error or unreliability. Accounting for error variance creates factors that are mathematically perfectly reliable, which increases the statistical power of the overall analysis.

SEM also allows researchers to test complex relationships between multiple factors and indicators at once, and it can accommodate multiple outcome $(\mathrm{Y})$ factors in one model. In addition, the fit of one model can also be compared with the fit of alternative models to determine which one best reflects the data. This process involves determining whether the chi-square value for the more restricted model is significantly different from the chisquare value for the less restrictive model by examining the statistical significance of the difference between the two chi-square values. 


\section{Specific Approach to Data Analyses}

We first examined the data for nonnormality to determine the appropriate estimator to use in the analyses. We then conducted a CFA to evaluate the validity of the fourfactor PTSD and one-factor combat exposure measurement model before proceeding with the SEM. Raw CAPS severity scores (frequency + intensity) for each CAPS item were analyzed as indicators of the four correlated PTSD factors reexperiencing, avoidance, emotional numbing, and hyperarousal. Raw scores on each of the seven items of the CES were used as indicators of the latent factor combat exposure. After determining the appropriateness of this measurement factors (using the fit statistics and cutoff values specified in the following paragraphs), we then included these factors in SEMs that examined the relationship between combat exposure, PTSD, and functioning.

The first SEM examined all possible direct and indirect effects by regressing the four latent PTSD factors on the latent factor combat exposure and by regressing the observed indicator GAF on the PTSD and combat exposure latent factors (the baseline model). Correlated disturbances among the PTSD factors were specified, because the four PTSD factors were expected to correlate with one another even after common variance attributed to combat exposure was removed from them. The second SEM removed the indirect effects by regressing GAF on combat exposure only (the direct effect model). The third SEM removed the direct effect from the baseline model by regressing the PTSD factors on combat exposure and by regressing GAF on the PTSD factors (the indirect effects model). We then examined the fit of a pared-down indirect effects model that included only the hypothesized associations (i.e., regressive paths from combat exposure to reexperiencing and avoidance and paths from emotional numbing to GAF).

To compare the relative fit of the baseline model with the subsequent indirect and direct effects models, we calculated the difference in the chi-square value between the baseline model and a given restricted model (e.g., the direct effect model). Next, we evaluated the statistical significance of the change in chi-square to see whether the change in chi-square exceeded the critical value of chi-square given the change in degrees of freedom (the critical value is obtained from a standard chi-square distribution table). Chi-square differences that exceed the critical value suggest that one model (typically the less restrictive baseline model) provides significantly better fit to the data. Chi-square differences that do not exceed the critical value suggest that the more restricted (nested) model provides a fit that is equivalent to the baseline model and is preferable, because it does so with greater parsimony than the baseline saturated model.

All analyses were conducted with the Mplus 3.11 statistical software (Muthén \& Muthén; Los Angeles, California) [32]. Model fit for the CFA and the SEMs were evaluated with fit statistics from the absolute fit, parsimony, and comparative fit classes of indices, following recommended guidelines and cutoff values [32-35]. In particular, from the absolute fit class, the chi-square value provided an index of the degree to which the model reproduced the sample variances and covariances. With this statistic, smaller values indicate better fit. In theory, the $p$-value for the chi-square should not be statistically significant, since this would indicate no significant difference between the predicted model and the observed relations among the data. In practice, however, the $p$-value for the chi-square will be significant in large samples even when relatively minor differences exist between the observed and predicted models. The standardized rootmean-square residual (SRMR), another fit statistic from the absolute fit class of indices, reflects the average difference between correlations in the observed data and correlations predicted by the model. SRMR values $<0.08$ indicate a good model fit. From the parsimony class of fit statistics, we used the root-mean-square error of approximation, which relies on the noncentral chi-square distribution and penalizes for model complexity. With this metric, values $<0.06$ indicate a good fit [34]. Finally, we also used the Tucker-Lewis index (TLI), or nonnormed fit index, which includes a penalty function for the addition of freely estimated parameters that do not markedly improve fit, and the comparative fit index (CFI), which evaluates the fit of a user-specified solution relative to a more restricted, nested baseline model. Both TLI and CFI are statistics from the comparative fit class that generally compares the fit of the specified model with the null model. With these indices, fit improves as values approach 1.0 , with values $>0.90$ considered adequate fit and values $>0.95$ considered good model fit.

\section{RESULTS}

Skewness and kurtosis tests for all variables (CAPS, $\mathrm{CES}$, and GAF) revealed that the data were generally normally distributed. Only two indicators yielded a skewness or kurtosis statistic greater than \pm 2 . The kurtosis values for CAPS items D1 and D4 were 3.34 and 2.37, 
respectively, but the skewness statistics for these items were within the acceptable range. Given the minimal evidence for nonnormality in the data, the analyses were conducted with the maximum likelihood estimator.

\section{Measurement Model of PTSD and Combat Exposure}

We then examined the fit of the measurement model in which the 17 CAPS items were specified to load onto four correlated PTSD factors and the 7 CES items were specified to load on the combat exposure factor. CAPS items B1 to B5 were loaded on the reexperiencing factor (with item B1 as the marker indicator of the factor), items $\mathrm{C} 1$ and $\mathrm{C} 2$ on the avoidance factor (with C1 the marker indicator), items $\mathrm{C} 3$ to $\mathrm{C} 7$ on the emotional numbing factor (with C4 the marker indicator), and items D1 to D5 on the hyperarousal factor (with D3 the marker indicator). CES item 1 was the marker indicator for the combat exposure factor. The four-factor model of PTSD and one-factor model of combat exposure fit the data well (Table 1), allowing us to proceed with the proposed SEM. Completely standardized factor loadings and indicator error for the measurement model are displayed in Table 2. All items loaded significantly on their respective factors, with the exception of C3 (psychogenic amnesia).

We also examined the bivariate correlations between each PTSD symptom factor and GAF. These associations are presented in Table $\mathbf{3}$ and show that each PTSD symptom factor was significantly related to GAF in the expected (negative) direction. (Associations between the combat and PTSD symptom latent factors are not presented because these values are identical to the structural paths presented in the Figure.)

*This result has been observed in previous studies [13] and should raise questions about the validity of this symptom as an indicator of PTSD.

\section{Structural Model of Combat Exposure, PTSD, and Functioning}

The baseline SEM, with direct and indirect paths of combat exposure and PTSD to GAF, fit the data well (Table 1). The second SEM, in which only the direct path from combat exposure to GAF was specified, provided a significantly poorer fit to the data than did the baseline model (Table 1) $\left(\Delta \chi^{2}(8)=78.91, p<0.05\right)$. The third SEM, in which only the indirect paths from combat exposure to GAF via PTSD were specified, fit the data better (Table 1) and did not damage model fit in comparison with the baseline model $\left(\Delta \chi^{2}(1)=0.29, p>0.05\right)$. The structural paths of this model are presented in Figure (a). In this model, combat exposure was significantly associated only with reexperiencing, while emotional numbing and avoidance were the only significant predictors of GAF. ${ }^{\dagger}$

We next examined the fit of our hypothesized model, which was a reduced version of the indirect effects model in which combat exposure predicted only reexperiencing and avoidance and the only predictor of GAF was emotional numbing. This model provided significantly poorer fit, as indexed by a significant chi-square difference test, than did the baseline model $\left(\Delta \chi^{2}(6)=19.73, p<0.05\right.$; Table 1 and Figure (b)). Given that the full indirect paths

\footnotetext{
${ }^{\dagger}$ The bivariate correlations in Table $\mathbf{3}$ show that hyperarousal was negatively associated with GAF and positively associated with the other PTSD symptom factors. This finding suggests that the large, yet nonsignificant, pathway from hyperarousal to GAF likely reflected a statistical suppressor effect. That is, after we controlled for overlapping positive variance between hyperarousal and emotional numbing and avoidance, the remaining variance in the hyperarousal to GAF path was nonsignificant and positive (either as a result of measurement error or of outlier cases in which a positive relationship existed between the two variables).
}

Table 1.

Model testing results for structural equation modeling of associations between combat exposure, posttraumatic stress disorder, and functioning.

\begin{tabular}{lcccccccc}
\hline \multicolumn{1}{c}{ Model } & $\chi^{\mathbf{2}}$ & $\boldsymbol{d} \boldsymbol{f}$ & SRMR & RMSEA & TLI & CFI & AIC & BIC \\
\hline Measurement & 375.69 & 246 & 0.07 & 0.04 & 0.92 & 0.93 & $29,065.63$ & $29,268.27$ \\
Direct and Indirect Effects & 375.17 & 261 & 0.05 & 0.04 & 0.93 & 0.94 & $31,291.41$ & $31,531.57$ \\
Direct Effects Only & 454.08 & 269 & 0.08 & 0.05 & 0.89 & 0.90 & $31,354.32$ & $31,564.46$ \\
Indirect Effects Only & 375.46 & 262 & 0.05 & 0.04 & 0.93 & 0.94 & $31,289.70$ & $31,526.11$ \\
Hypothesized & 394.90 & 267 & 0.05 & 0.04 & 0.92 & 0.93 & $31,299.14$ & $31,516.79$ \\
Final & 383.35 & 267 & 0.05 & 0.04 & 0.93 & 0.94 & $31,287.59$ & $31,505.24$ \\
\hline
\end{tabular}

$\overline{\mathrm{AIC}}=$ Akaike information criterion, BIC = Bayesian information criterion, CFI = comparative fit index, $d f=$ degrees of freedom, RMSEA = root-mean-square error of approximation, SRMR = standardized root-mean-square residual, TLI = Tucker-Lewis index. 
Table 2.

Measurement model of postraumatic stress disorder (PTSD) and combat exposure: Indicator loadings (and unreliability).

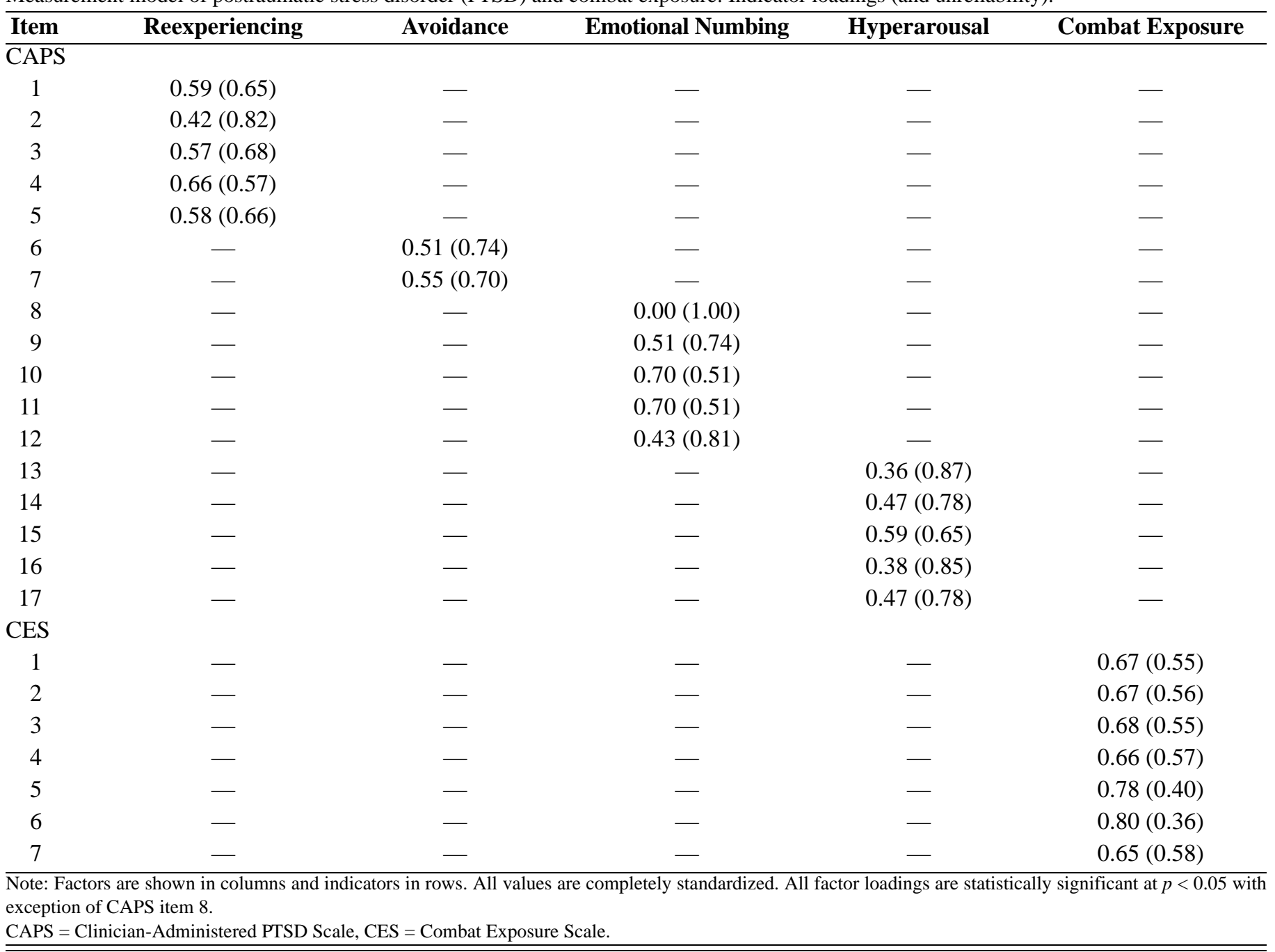

Table 3.

Bivariate correlations among posttraumatic stress disorder latent factors and Global Assessment of Functioning (GAF).

\begin{tabular}{lccccc}
\hline \multicolumn{1}{c}{ Factor } & $\mathbf{1}$ & $\mathbf{2}$ & $\mathbf{3}$ & $\mathbf{4}$ & $\mathbf{5}$ \\
\hline 1. Reexperiencing & - & - & - & - & - \\
2. Avoidance & $0.55^{*}$ & - & - & - & - \\
3. Emotional Numbing & $0.35^{*}$ & $0.38^{*}$ & - & - & - \\
4. Hyperarousal & $0.71^{*}$ & $0.57^{*}$ & $-0.40^{*}$ & $-0.31^{*}$ & - \\
5. GAF & $-0.30^{*}$ & $-0.41^{*}$ & & \\
\hline${ }^{*} p<0.05$. & & & \\
\hline
\end{tabular}

model fit the data as well as the baseline model and was more parsimonious, the indirect model was retained as the best-fitting model.

As shown in Figure (a), not all paths in the bestfitting model were statistically significant, so we system- atically removed nonsignificant paths (one at a time) to arrive at a more parsimonious model. We did so by using the chi-square difference testing approach to systematically evaluate whether removing a path damaged overall fit. Decisions regarding which paths to eliminate were 
also based on the extent to which a given path failed to reach statistical significance and hypotheses concerning the expected associations between the factors. We determined statistical significance of a pathway or factor loading by dividing the unstandardized parameter estimate by the standard error, yielding a $Z$ statistic for which values greater than \pm 1.96 are significant at $p<0.05$. This process of eliminating the nonsignificant paths led to a final model in which reexperiencing was the only factor regressed on combat exposure and GAF was regressed on only the avoidance and emotional numbing factors.

Table 1 displays the fit statistics for the respecified final model, which did not damage model fit in comparison with the original less parsimonious model $\left(\Delta \chi^{2}(6)=8.18\right.$, $p>0.05$ ). Figure (c) displays the unstandardized parameter estimates (e.g., in the original metric of the measures), $Z$ statistics, and completely standardized parameter estimates for the final model. The SEM revealed that combat exposure was a significant predictor of reexperiencing symptoms but of no other PTSD factor (completely standardized path coefficient $=0.24$ ). Emotional numbing was a significant predictor of functioning (completely standardized path coefficient $=-0.26$ ), as was avoidance (completely standardized path coefficient $=-0.32$ ).

\section{DISCUSSION}

This study examined the differential associations between the severity of combat exposure (CES scores), four symptom factors of PTSD, and global functioning (GAF scores) in a sample of male veterans receiving services at a VA PTSD clinic. We advanced three primary hypotheses. First, the association between combat exposure and GAF would be fully mediated by PTSD. Second, combat exposure intensity would be more highly correlated with the severity of reexperiencing and avoidance symptoms than with the emotional numbing and hyperarousal symptoms. Third, emotional numbing would be more strongly associated with GAF than symptoms of the other three factors.

We tested the first hypothesis by comparing the relative fit of models that either did or did not include a direct path from combat exposure to global functioning. As hypothesized, results indicated that a direct path was not necessary for an optimally fitting and parsimonious model. This finding implies that the association between combat exposure and global functional impairment was fully

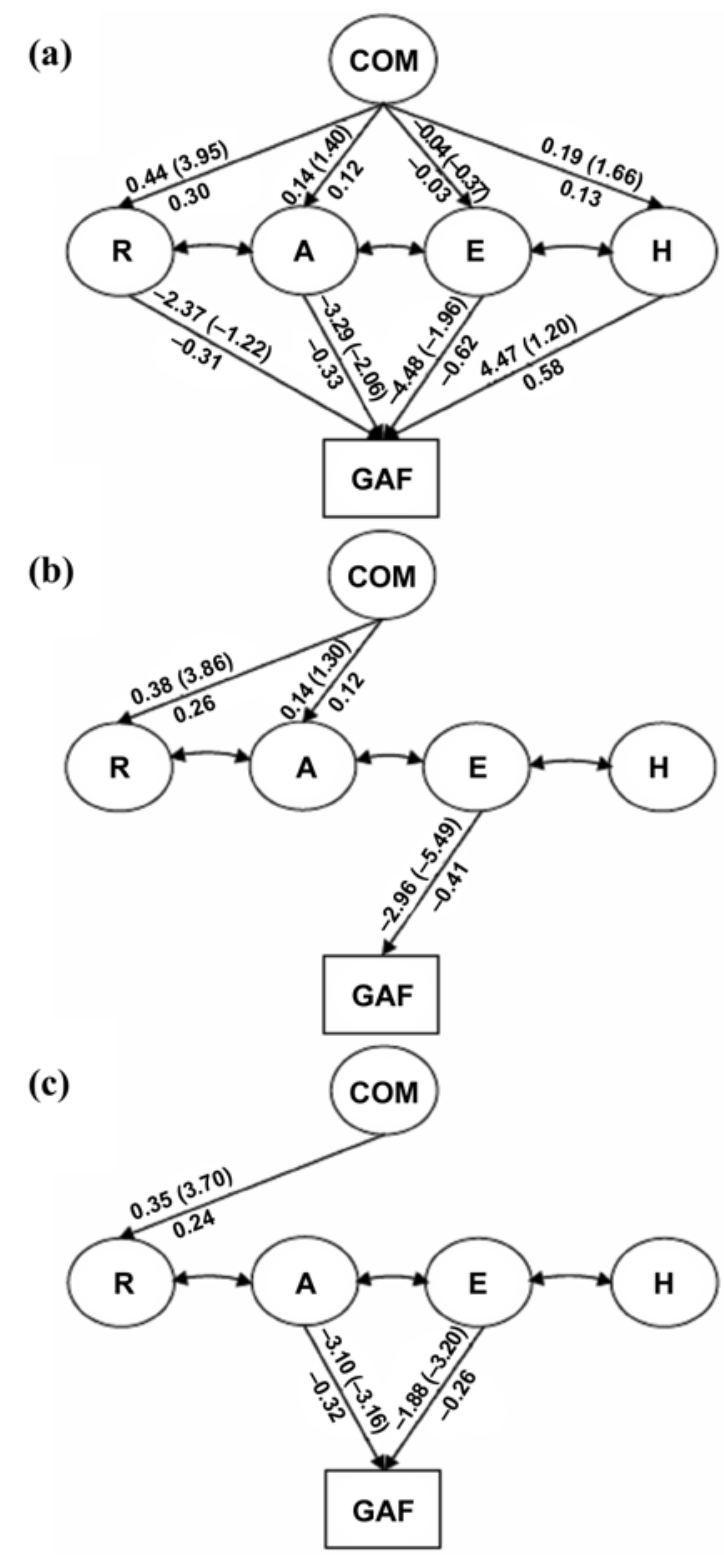

Figure.

Results from (a) indirect paths, (b) hypothesized, and (c) final accepted models. First value on each path is unstandardized path coefficient, followed by critical ratio (z-test) in parentheses. $Z$ statistics greater than or equal to \pm 1.96 indicate that pathway was statistically significant at $p<$ 0.05 . Value below each pathway is completely standardized coefficient. Only structural paths are shown in model (indicator loadings and reliabilities are provided in Table 2). To simplify presentation and avoid redundancy, only a subset of posttraumatic stress disorder (PTSD) factor correlations (denoted by curved double-headed arrows) is shown to illustrate their interrelationships; actual statistical model included all possible PTSD factor correlations and/or correlations among factor disturbances where applicable. None of these correlations differed from factor correlations presented in Table 3 by more than $r=0.04$. A = avoidance, $\mathrm{COM}=$ combat exposure, $\mathrm{E}=$ emotional numbing, $\mathrm{GAF}=$ Global Assessment of Functioning, $\mathrm{H}=$ hyperarousal, $\mathrm{R}$ = reexperiencing. 
mediated by PTSD severity, which supports the role of PTSD as a mechanism for the association between combat exposure and subsequent global functional impairment.

The best-fitting model included a significant regressive path from combat exposure to reexperiencing symptoms. This result provided partial support for our second hypothesis that combat exposure would be more strongly linked with reexperiencing and avoidance than with the other two factors. Prior research and theory suggest that avoidance symptoms are functionally intertwined with reexperiencing because they minimize exposure to traumarelated stimuli and the intense states of reexperiencing that they evoke [36-37]. Consistent with this, our results revealed a strong correlation $(0.55)$ between these two factors. However, the unique effect of combat exposure on reexperiencing points to the primacy of these symptoms in the psychopathology of the disorder. To the extent that CES scores can be conceptualized as a causal variable in this model, these findings also suggest that trauma exerts its most direct etiologic effect on the development of reexperiencing symptoms.

The CES measures the frequency and intensity of potentially traumatic experiences endured during the course of a war zone deployment. Results of this study suggest that as the number of trauma exposures increased in this sample, the severity of reexperiencing symptoms increased in a linear fashion, i.e., an additive effect. This finding is relevant to planning for the mental health needs of veterans of the current conflicts in Iraq and Afghanistan, for whom tour lengths are being extended and multiple deployments have become increasingly common. The implication is that as deployments are extended, the risk for exposure to multiple potentially traumatic events increases. This, in turn, can be expected to increase the likelihood and severity of PTSD and associated functional impairment in veterans.

Of the PTSD symptom factors, we had hypothesized on the basis of prior research that the numbing symptoms would be most strongly associated with GAF. Results showed support for this hypothesis, with the best-fitting model including significant regressive paths from numbing symptoms to GAF. An unexpected result was the finding of an additional significant path from effortful avoidance to GAF. These findings suggest that symptoms that involve restricted freedom of action, reduced pleasure and interest in activities, and interference in social engagement accounted for the largest proportion of variance in global functioning.
One important caveat to our conclusions regarding the relationship of PTSD symptomatology to global functioning is that avoidance and numbing symptoms together accounted for only 17 percent of the variance in GAF scores. This estimate was likely attenuated by error in the measurement of GAF, a scale that possesses modest interrater and test-retest reliability in this population [10-11]. Other likely sources of unexplained variance in GAF scores included physical illness or disability and comorbid psychopathology that were not modeled in these analyses. With respect to the latter, of the anxiety disorders, PTSD shows perhaps the most severe and diverse pattern of diagnostic comorbidity [38] with frequently co-occurring conditions ranging from those of the internalizing spectrum (i.e., the anxiety and unipolar mood disorders) to the externalizing spectrum (i.e., antisociality and substance dependence). Prior research suggests an additive effect of comorbidity on functional impairment, with comorbid patients reporting more severe symptoms and greater impairment than patients who only meet criteria for a single disorder. Future studies should examine the role that these important factors play in producing functional impairment.

To our knowledge, this study was the first to use SEM to examine how combat exposure and GAF relate to the individual PTSD symptom factors. The ability to model the latent factor structure of PTSD, partition true variance from error variance, and evaluate each symptom factor as a mediator of the relationship between combat exposure and GAF were strengths of the data analysis. The large clinical sample and reliance upon a gold standard diagnostic interview for the assessment of PTSD were additional strengths. Study limitations included, first, the fact that many veterans in the sample were seeking disability compensation for PTSD and this may have led to biased (elevated) symptom reporting (although how this would have produced the differential relationships that we observed across PTSD symptom factors is unclear). Second, we were unable to examine temporal relationships between variables because of the cross-sectional nature of the data. Third, reports of past combat exposure were possibly biased by factors that were confounded with PTSD symptomatology, as previously shown [39]. Fourth, in this study, as in any study of the association between measures of symptomatology and ratings of global functioning, the problem of criterion contamination exists, because the severity of PTSD symptoms is indexed, at least in part, by a judgment of the degree to which each symptom produces functional impairment, an unavoidable conceptual overlap exists between the 
predictor and outcome variables. Fifth, because data were drawn from an archive based on routine clinical assessments, no index of the interrater reliability of these measures was available. Finally, the sample was exclusively male veterans who served primarily during the Vietnam war, rendering the generalizability of these results to other PTSD samples open to question.

\section{CONCLUSIONS}

Results of this study should raise concern about the VA's reliance on the GAF as a benchmark for the assessment of PTSD-related functional impairment. Results revealed modest associations between PTSD symptomatology and GAF scores, and these associations were limited to the effortful avoidance and numbing symptoms. If VA C\&P determinations are to be based on the level of functional impairment produced by PTSD, then multidimensional measures of impairment, disability, and quality of life should be incorporated into assessment strategies so that the many important domains of functioning that can be adversely affected by the disorder are evaluated. Additional attention to the psychometric properties of instruments used in the measurement and evaluation of PTSD is warranted, and applying modern psychometric theory to the development of more sophisticated, reliable, and valid measures of military-related disorders and functional disability should become a priority for future research [40].

\section{ACKNOWLEDGMENTS}

Elaine Martin is now with the Department of Psychology, University of Nebraska-Lincoln.

This material was based on work supported by a VA Merit Review grant to Mark W. Miller.

The authors have declared that no competing interests exist.

\section{REFERENCES}

1. Kulka RA, Schlenger WE, Fairbank JA, Hough RL, Jordan BK, Marmar CR, Weiss DS. Trauma and the Vietnam war generation: Report of findings from the National Vietnam Veterans Readjustment Study. New York (NY): Brunner/ Mazel; 1990.
2. Dohrenwend BP, Turner JB, Turse NA, Adams BG, Koenen KC, Marshall R. The psychological risks of Vietnam for U.S. veterans: A revisit with new data and methods. Science. 2006;313(5789):979-82. [PMID: 16917066]

3. Hoge CW, Castro CA, Messer SC, McGurk D, Cotting DI, Koffman RL. Combat duty in Iraq and Afghanistan, mental health problems, and barriers to care. N Engl J Med. 2004; 351(1):13-22. [PMID: 15229303$]$

4. Department of Veterans Affairs Office of Inspector General. Review of state variances in VA disability compensation payments. Report No. 05-00765-137. Washington (DC): VA Office of the Inspector General; 2005. Available from: http:// www.va.gov/oig/52/reports/2005/VAOIG-05-00765-137.pdf

5. American Psychiatric Association. Diagnostic and statistical manual of mental disorders. 4th ed. Washington (DC): American Psychiatric Association; 1994.

6. Institute of Medicine. Posttraumatic stress disorder: Diagnosis and assessment. Washington (DC): National Academies Press; 2006.

7. Department of Veterans Affairs. VHA Directive 97-059: Instituting Global Assessment of Function (GAF) scores in axis V for mental health patients. Washington (DC): Veterans Health Administration; 1997. Available from: http://www.avapl.org/gaf/gaf.html

8. Roy-Byrne P, Dagadakis C, Unutzer J, Ries R. Evidence for limited validity of the revised global assessment of functioning scale. Psychiatr Serv. 1996;47(8):864-66. [PMID: 8837160]

9. Greenberg GA, Rosenheck RA. Using the GAF as a national mental health outcome measure in the Department of Veterans Affairs. Psychiatr Serv. 2005;56(4):420-26. [PMID: 15812091]

10. Miller MW, Greif JL, Smith AA. Multidimensional Personality Questionnaire profiles of veterans with traumatic combat exposure: Externalizing and internalizing subtypes. Psychol Assess. 2003;15(2):205-15. [PMID: 12847781]

11. Miller MW, Kaloupek DG, Dillon AL, Keane TM. Externalizing and internalizing subtypes of combat-related PTSD: A replication and extension using the PSY-5 scales. J Abnorm Psychol. 2004;113(4):636-45. [PMID: 15535795]

12. Asmundson GJ, Stapleton JA, Taylor S. Are avoidance and numbing distinct PTSD symptom clusters? J Trauma Stress. 2004;17(6):467-75. [PMID: 15730065$]$

13. Palmieri PA, Weathers FW, Difede J, King DW. Confirmatory factor analysis of the PTSD Checklist and the ClinicianAdministered PTSD Scale in disaster workers exposed to the World Trade Center Ground Zero. J Abnorm Psychol. 2007; 116(2):329-41. [PMID: 17516765$]$

14. Simms LJ, Watson D, Doebbeling BN. Confirmatory factor analyses of posttraumatic stress symptoms in deployed and nondeployed veterans of the Gulf War. J Abnorm Psychol. 2002;111(4):637-47. [PMID: 12428777]

15. Watson D, Gamez W, Simms LJ. Basic dimensions of temperament and their relation to anxiety and depression: A symptom-based perspective. J Res Pers. 2005;39(1):46-66. 
16. Anthony JL, Lonigan CJ, Hecht SA. Dimensionality of posttraumatic stress disorder symptoms in children exposed to disaster: Results from confirmatory factor analyses. J Abnorm Psychol. 1999;108(2):326-36. [PMID: 10369043]

17. Lonigan CJ, Shannon MP, Taylor CM, Finch AJ Jr, Sallee FR. Children exposed to disaster: II. Risk factors for the development of post-traumatic symptomatology. J Am Acad Child Adolesc Psychiatry. 1994;33(1):94-105. [PMID: 8138526]

18. Kimerling R, Clum GA, Wolfe J. Relationships among trauma exposure, chronic posttraumatic stress disorder symptoms, and self-reported health in women: Replication and extension. J Trauma Stress. 2000;13(1):115-28. [PMID: 10761178]

19. Palmieri PA, Fitzgerald LF. Confirmatory factor analysis of posttraumatic stress symptoms in sexually harassed women. J Trauma Stress. 2005;18(6):657-66. [PMID: 16382424]

20. Norris R, Maguen S, Litz B, Adler A, Brett T. Physical symptoms in peacekeepers: Has the role of deployment stress been overrated? Stress Trauma Crisis Int J. 2005;8:251-65.

21. Marshall RD, Turner JB, Lewis-Fernadez R, Koenen K, Neria Y, Dohrenwend BP. Symptom patterns associated with chronic PTSD in male veterans: New findings from the National Vietnam Veterans Readjustment Study. J Nerv Ment Dis. 2006;194(4):275-78. [PMID: 16614549]

22. North CS, Nixon SJ, Shariat S, Mallonee S, McMillen JC, Spitznagel EL, Smith EM. Psychiatric disorders among survivors of the Oklahoma City bombing. JAMA. 1999: 282(8):755-62. [PMID: 10463711]

23. Solomon Z, Mikulincer M. Posttraumatic intrusion, avoidance, and social functioning: A 20-year longitudinal study. 2007;75(2):316-24. [PMID: 17469889]

24. Johnson DM, Palmieri PA, Jackson AP, Hobfoll SE. Emotional numbing weakens abused inner-city women's resiliency resources. J Trauma Stress. 2007;20(2):197-206. [PMID: 17427905$]$

25. McFarlane AC, Atchinson M, Rafalowicz E, Papay P. Physical symptoms in post-traumatic stress disorder. J Psychosom Res. 1994;38(7):715-26. [PMID: 7877126]

26. Beckham JC, Crawford AL, Feldman ME, Kirby AC, Hertzberg MA, Davidson JR, Moore SD. Chronic posttraumatic stress disorder and chronic pain in Vietnam combat veterans. J Psychosom Res. 1997;43(4):379-89. [PMID: 9330237]

27. Blake DD, Weathers FW, Nagy LM, Kaloupek DG, Klauminzer G, Charney DS, Keane T. A clinician rating scale for assessing current and lifetime PTSD: The CAPS-1. Behav Therapist. 1990;13:187-88.
28. Weathers FW, Keane TM, Davidson JR. Clinicianadministered PTSD scale: A review of the first ten years of research. Depress Anxiety. 2001;13(3):132-56.

[PMID: 11387733]

29. Keane TM, Fairbank JA, Caddell JM, Zimering RT, Taylor KL, Mora CA. Clinical evaluation of a measure to assess combat exposure. Psychol Assess. 1989;1:53-55.

30. Keane TM, Brief DJ, Pratt EM, Miller MW. Assessment of PTSD and its comorbidities in adults. In: Friedman MJ, Keane TM, Resick PA, editors. Handbook of PTSD: Science and practice. New York (NY): Guilford Press; 2007. p. 279-305.

31. Weathers FW, Ruscio AM, Keane TM. Psychometric properties of nine scoring rules of the Clinician-Administered Posttraumatic Stress Disorder Scale. Psychol Assess. 1999; 11(2):124-33.

32. Muthén LL, Muthén B. Mplus: Statistical analysis with latent variables: User's guide. 4th ed. Los Angeles (CA): Muthén \& Muthén; 2007.

33. Brown TA. Confirmatory factor analysis for applied research. New York (NY): Guilford Press; 2006.

34. Hu L, Bentler PM. Cutoff criteria for fit indexes in covariance structure analysis: Conventional criteria versus new alternatives. Struct Equation Model. 1999;6(1):1-55.

35. Kline RB. Principles and practice of structural equation modeling. 2nd ed. New York (NY): Guilford; 2005.

36. Keane TM, Zimering RT, Caddell JM. A behavioral formulation of posttraumatic stress disorder in Vietnam veterans. Behav Therapist. 1985;8(1):9-12.

37. Keane TM, Barlow DH. Posttraumatic stress disorder. In: Barlow D, editor. Anxiety and its disorders: The nature and treatment of anxiety and panic. 2nd ed. New York (NY): Guilford Press; 2002. p. 418-53.

38. Brown TA, Campbell LA, Lehman CL, Grisham JR, Mancill RB. Current and lifetime comorbidity of the DSM-IV anxiety and mood disorders in a large clinical sample. J Abnorm Psychol. 2001;110(4):585-99. [PMID: 11727948]

39. Koenen KC, Stellman SD, Dohrenwend BP, Sommer JF Jr, Stellman JM. The consistency of combat exposure reporting and course of PTSD in Vietnam War veterans. J Trauma Stress. 2007;20(1):3-13. [PMID: 17345644]

40. Weathers FW, Keane TM, King LA, King DW. Psychometric theory in the development of posttraumatic stress disorder assessment tools. In: Wilson JP, Keane TM, editors. Assessing psychological trauma and PTSD. 4th ed. New York (NY): Guilford Press; 1997. p. 98-135.

Submitted for publication June 15, 2007. Accepted in revised form November 14, 2007. 
